
\title{
25 Research Square \\ Phosphoglucomutase 1 Contributes to Optimal Cyst Development in Toxoplasma Gondii
}

\section{Emily V. Quach}

Laney College

\section{Binh Cao}

University of California San Francisco

\section{Edres Babacarkhial}

California State University East Bay

\section{Daniel Ho}

Stanford University School of Medicine

\section{Janak Sharma}

California State University East Bay

Pascale S. Guiton ( $\sim$ pascale.guiton@csueastbay.edu )

California State University East Bay https://orcid.org/0000-0002-8186-3515

\section{Research Article}

Keywords: phosphoglucomutase, glycolysis, gluconeogenesis, Toxoplasma, amylopectin, tissue cysts, stage conversion

Posted Date: February 16th, 2022

DOI: https://doi.org/10.21203/rs.3.rs-1191933/v1

License: (c) (i) This work is licensed under a Creative Commons Attribution 4.0 International License.

Read Full License 


\section{Abstract}

Objective: Toxoplasma gondii is a ubiquitous parasite of medical and veterinary importance; however, there exists no cure for chronic toxoplasmosis. Metabolic enzymes required for the production and maintenance of tissue cysts represent promising targets for novel therapies. Here, we use reverse genetics to investigate the role of Toxoplasma phosphoglucomutase 1, PGM1, in Toxoplasma growth and cystogenesis.

Results: We found that disruption of pgm1 did not significantly affect Toxoplasma intracellular growth and the lytic cycle. pgm1-defective parasites could differentiate into bradyzoites and produced cysts containing amylopectin in vitro. However, cysts produced in the absence of pgm 1 were significantly smaller than wildtype. Together, our findings suggest that PGM1 is dispensable for in vitro growth but contributes to optimal Toxoplasma cyst development in vitro, thereby necessitating further investigation.

\section{Introduction}

Toxoplasma gondii is an obligate intracellular protozoan responsible for toxoplasmosis in humans and other warm-blooded animals. Infections occur mostly from consuming contaminated water, food, or undercooked meat from chronically infected animals [1]. Bradyzoites inside tissue cysts are released into the gastrointestinal tract where they invade enterocytes and convert to tachyzoites inside a parasitophorous vacuole (PV). Tachyzoites replicate rapidly, eventually lysing out of the host cell to disseminate throughout the body. In response to stressful stimuli, they convert back to bradyzoites which remain encysted in the brain and skeletal muscles for life [2]. Chronic toxoplasmosis is incurable and parasite reactivation life-threatening, particularly for the immunocompromised [3].

Bradyzoites are replete with cytoplasmic amylopectin granules [4]. The tight regulation of enzymes involved in metabolizing this polysaccharide is critical for tissue cyst production and survival [5-8]. Phosphoglucomutases (PGMs) catalyze the interconversion of glucose-1-phosphate to glucose-6phosphate [9], effectively linking amylopectin metabolism to glycolysis in this parasite. Both PGM paralogs in Toxoplasma [10], PGM1, also known as parafusin-related Toxoplasma protein 1 (PRP1) [11], and PGM2, are upregulated during chronic infection in mice [12] and have been implicated in calcium $\left(\mathrm{Ca}^{2+}\right)$-dependent signaling for microneme secretion [13-15].

Here, we used the CRISPR/Cas9 gene-editing system [16] to disrupt pgm 1 in a cyst-forming Toxoplasma strain. Our data show that this mutation did not prevent intracellular replication or the completion of the lytic cycle. While both strains could produce amylopectin-containing cysts, we found that pgm1-defective cysts are significantly smaller than the parent's. Together, our findings corroborate previous reports that PGM1 is dispensable for Toxoplasma viability and demonstrate that the enzyme contributes to optimal cyst development in vitro.

\section{Materials And Methods}




\section{Parasite and host cells}

Human foreskin fibroblasts (HFFs) and Me49 $\Delta$ xxgprt, a Type II strain of Toxoplasma lacking hypoxanthine-xanthine-guanine phosphoribosyltransferase (HXGPRT), were kind gifts from John Boothroyd at Stanford University. Parasites were maintained in HFFs in Dulbecco's modified Eagle medium supplemented with $10 \%$ fetal bovine serum, $2 \mathrm{mM} \mathrm{L-glutamine,} 2.5 \mu \mathrm{g} / \mathrm{ml}$ fungizone, $100 \mathrm{U} / \mathrm{ml}$ penicillin, and $100 \mu \mathrm{g} / \mathrm{ml}$ streptomycin (CDMEM) at $37^{\circ} \mathrm{C}$ and $5 \% \mathrm{CO}_{2}$.

\section{Disruption of pgm1}

All primers used in this study are listed in Additional file 1. pSAG::Cas9-U6::sgPGM1 was obtained by substitution of sgUPRT with sgPGM1 in pSAG1::Cas9-U6::sgUPRT [16] using Q5 site-directed mutagenesis (New England Biolabs Inc, NEB). pUC19 modified to express hxgprt under the dihydrofolate reductase (DHFR) promoter using standard molecular cloning techniques to create pDHFR:: hxgprt. Freshly released Me49 $\Delta$ hxgprt (WT) were transfected with pSAG1::Cas9-U6::sgUPRT and linearized pDHFR:: hxgprt at a 1:3 molar ratio in a $4 \mathrm{~mm}$ gap cuvette in an BTX ECM 630 Exponential Decay Wave electroporator system (BTX Harvard Apparatus) [16]. Transgenic Me49 $\Delta$ hxgprt $\Delta p g m 1$ parasites ( $\Delta p g m 1)$ were obtained after 10 days of selection in CDMEM containing $25 \mu \mathrm{g} / \mathrm{ml}$ of mycophenolic acid and 50 $\mu \mathrm{g} / \mathrm{ml}$ xanthine and cloned by limiting dilutions [17]. Disruption of $p g m 1$ and integration of the selection cassette were confirmed by polymerase chain reaction (PCR) and DNA sequencing (Elim Biopharmaceuticals Inc).

\section{Replication assay}

Freshly released parasites were centrifuged at $1500 \mathrm{rpm}$ for 10 minutes and washed once with 1 XPBS. Confluent HFFs on glass coverslips were infected with $1.2 \times 10^{5}$ parasites in CDMEM for $24 \mathrm{~h}$. The number of parasites per vacuole was determined by immunofluorescence microscopy.

\section{Plaque assay}

WT and $\Delta p g m 1$ tachyzoites were syringe-lysed through a $27 \mathrm{G}$ needle and passed through a $5 \mu \mathrm{m}$ filter. Confluent HFFs were infected with 250 parasites in CDMEM and incubated at $37^{\circ} \mathrm{C}$ with $5 \% \mathrm{CO}_{2}$ for 10 days undisturbed. Following methanol fixation and crystal violet staining, plaque numbers and sizes were determined using a stereoscope (Leica EZ4) and ImageJ version 1.52A (National Institutes of Health).

\section{Tachyzoite-to-bradyzoite differentiation}

Tachyzoites were induced to differentiate into bradyzoites in HFFs as previously described [18]. Briefly, confluent HFFs on glass coverslips were infected with $4.8 \times 10^{4}$ parasites for 3 hours in cDMEM before replacing the medium with Switch Medium (RPMI 1640 supplemented with $1 \%$ fetal bovine serum, 100 $\mathrm{U} / \mathrm{ml}$ penicillin, and $100 \mu \mathrm{g} / \mathrm{ml}$ streptomycin, $10 \mathrm{mg} / \mathrm{mL}$ HEPES, $\mathrm{pH}$ 8.2). Parasites were incubated for 4 
days at $37^{\circ} \mathrm{C}$ with ambient $\mathrm{CO}_{2}$ and the medium was changed every 24 hours to maintain alkaline conditions.

\section{Immunostaining fluorescence assay and amylopectin staining}

Infected monolayers were washed with phosphate-buffered saline (PBS) and fixed with 4\% paraformaldehyde (Electron Microscopy Sciences) for 15 minutes at room temperature (RT). Cells were permeabilized with $0.2 \%$ or $0.4 \%$ Triton $\mathrm{X}-100$ for 20 minutes and incubated for 1 hour in $3 \%$ Bovine Serum Albumin (BSA; Fisher Scientific) in PBS. Primary antibodies diluted in 3\% BSA/PBS (mouse aSAG1 1:10000, rabbit a-GRA7 1:1000) were added to the monolayers, when indicated, and incubated overnight at $4^{\circ} \mathrm{C}$. Unbound antibodies were washed away with three 5 -minute washes in 1 XPBS. The cells were then stained with secondary antibodies in 3\% BSA/PBS (Goat a -Mouse 546 or Goat a-Rabbit 488 at 1:5000) for 45 min at RT. Dolichos biflorus Agglutinin (DBA; Vector Laboratories) was used at 1:100 to detect the cyst wall. After washing as described above, the coverslips were mounted with VECTASHIELD Mounting Medium containing DAPI (Vector Laboratories). Amylopectin was stained with Periodic Acid Schiff (PAS; Fisher Scientific) according to the manufacturer's guidelines.

Immunofluorescence images were obtained using an inverted microscope (Leica DM IL LED) with 100X oil immersion objective. The number of parasites per vacuole and cyst areas, both selected from random fields of view, were determined using ImageJ version 1.52A and 1.53, respectively.

\section{Statistical methods}

Statistical analyses were performed using GraphPad Prism version 8.4.3. A $p$-value $\leq 0.05$ was considered statistically significant difference between groups.

\section{Results}

\section{Toxoplasma phosphoglucomutases are upregulated during chronic infection in mice}

Comparative transcriptomic and proteomic analyses [19] revealed that Toxoplasma expresses stagespecific proteins which enable the parasite to survive and to be efficiently transmitted between hosts. We mined the transcriptional data from Pittman et al. [12] available on the Toxoplasma Informatics Resources database (ToxoDB) [10] to specifically identify metabolic enzymes involved in gluconeogenesis and glycolysis that are significantly upregulated at least 2 folds in chronic vs. acute infection. Of the 422 genes upregulated in chronic infection, our analysis revealed 21 that are specifically associated with carbohydrate metabolism (Fig. 1A-B, Additional File 1). As expected, these genes include well-known glycolytic isoenzymes involved in tissue cyst formation, such as lactate dehydrogenase 2 (Idh2) [20] and enolase 1 (eno1) [21]. Interestingly, unlike /dh1/ldh2 and eno1/eno2 which are expressed in a stage-dependent manner, both PGM isoforms (pgm1 and pgm2) were upregulated 6.4 and 3.1 folds, respectively, in the chronic stage, 28 days post-infection (dpi) [12]. Transcriptional analyses of gene expression at 28, 90, and 120 dpi from Garfoot et al. [22] indicate that unlike pgm2 whose expression 
remained similar up to $120 \mathrm{dpi}$, pgm 1 transcripts further increased from 28 to $120 \mathrm{dpi}$. Together, this analysis strongly suggests that transcriptional regulation of $p g m 1 / p g m 2$ may be critical for the development and/or maintenance of tissue cysts in mice.

\section{Disruption of pgm1 does not hinder parasite growth in vitro}

To determine the contribution of PGM1 to Toxoplasma growth, we used the CRISPR-Cas9 gene-editing system to create an insertional mutant Me49 $\Delta h x g p r t \Delta p g m 1(\Delta p g m 1)$ by introducing a $h x g p r t$ selection cassette at the pgm 1 locus [16] (Fig. 2A-B). We assessed the intracellular growth of $\Delta p g m 1$ parasites vs. WT 24 hours after infection of HFFs in glucose replete growth medium. SAG1-positive parasites inside GRA7-positive vacuoles were enumerated. We found similar numbers of $\Delta p g m 1$ vacuoles with either 2, 4, or $\geq 8$ parasites as WT (Fig. 2C). Likewise, no significant differences in plaque numbers and sizes were observed 10 days after infection (Fig. 2D-E). Thus, as previously reported for Toxoplasma RH strain [15, 23], our data indicate that PGM1 is dispensable for Toxoplasma intracellular growth and lytic cycle in vitro, albeit in glucose-rich conditions.

\section{pgm1- defective parasites produced smaller amylopectin-containing cysts in vitro}

Given the upregulation of pgm1 in chronic infection, we tested whether disruption of pgm1 would impede tissue cyst formation. We induced tachyzoites to differentiate into bradyzoites in nutrient-poor, alkaline conditions in ambient $\mathrm{CO}_{2}$ [18]. After four days, we stained the monolayers with Dolichos biflorus agglutinin (DBA) to detect the cyst wall and Periodic Acid Schiff (PAS) to visualize amylopectin [8]. Both WT and $\Delta p g m 1$ parasites produced PAS-positive cysts (Fig. 3A), suggesting that PGM1 is not essential for amylopectin accumulation during stage conversion in vitro. However, further studies are required to determine any differences in the relative amount of this polysaccharide between WT and $\Delta p g m 1$ cysts. Interestingly, $\Delta p g m 1$ cysts were on average $\sim 4060$ pixels $^{2}$ smaller than WT ( $p=0.0362$ by Mann-Whitney test, Fig. $3 C$ ). Together, our results indicate that although PGM1 is not required for stage conversion and amylopectin storage, the enzyme contributes to optimal cyst development in vitro.

\section{Discussion}

PGM1 is one of two PGM isoforms differentially expressed in Toxoplasma [10,12, 24]. In this study, we showed that disruption of pgm1 in a cyst-forming Toxoplasma strain did not prevent intracellular growth or completion of the lytic cycle in glucose-replete conditions, corroborating previous studies in non-cyst forming Type I tachyzoites [15, 23]. Our observation that tachyzoites lacking pgm1 could differentiate into bradyzoites in the absence of glucose further supports the nonessential role of PGM1 and PGM1dependent glucose-6-phosphate production in tachyzoites as suggested by Imada et al. [25]. Interestingly, PGM1 has been implicated in $\mathrm{Ca}^{2+}$-dependent microneme secretion in tachyzoites $[11,13,15]$, and thus, like functionally characterized PGMs in other organisms [9, 26], it may play an unconventional role during Toxoplasma development. 
Although glycolysis is not required for tachyzoite viability, it is critical for tissue cyst formation and pathogenesis in mice [27]. Parasites lacking hexokinase, the first enzyme in glycolysis that catalyzes the phosphorylation of glucose to glucose-6-phosphate, produce smaller cysts in vitro [27]. This phenotype was recapitulated in pgm 1-defective parasites, further supporting the importance of glycolytic intermediates during cystogenesis. However, the absence of pgm1 did not abrogate amylopectin biosynthesis and storage, probably due to functional compensation with PGM2. While both pgm 1 and pgm2 transcripts are higher in bradyzoites than tachyzoites [24], the proteins share only $25 \%$ homology. PGM2 has a significantly lower enzymatic activity than PGM1 [25]. Interestingly, Saha et al. [15] demonstrated that PGM2 didn't compensate for the deletion of PGM1 in the context of $\mathrm{Ca}^{2+}$-regulated microneme secretion in tachyzoites.

Overall, this study suggests that PGM1 is not critical for Toxoplasma growth and differentiation; however, it is required for optimal cyst maturation. Future studies is needed to parse out the interplay and diverse activities of Toxoplasma PGMs and understand how they affect central carbon metabolism and developmental differentiation in this ubiquitous parasites.

\section{Limitations}

Due to institutional infrastructure failures that resulted in the loss of all parasite lines, including the ones used here, we were unable to perform complementation studies or growth assays in the presence or absence of various carbon sources. We also did not quantify PAS staining to identify any difference in amylopectin accumulation between WT and mutant parasites.

\section{Abbreviations}

BSA: Bovine Serum Albumin

DBA: Dolichos biflorus agglutinin

DHFR: dihydrofolate reductase

HFF: Human foreskin fibroblast

HXGPRT: hypoxanthine-xanthine-guanine phosphoribosyltransferase

PAS: Periodic Acid Schiff

PBS: phosphate-buffered saline

PCR: Polymerase Chain Reaction

PGM: Phosphoglucomutase

PV: parasitophorous vacuole 
RT: room temperature

WT: Wildtype strain

\section{Declarations}

\section{Ethics approval and consent to participate}

Not applicable

\section{Consent for publication}

Not applicable

\section{Availability of data and materials}

The transcriptional dataset used in this study is publicly available on ToxoDB version 54 (www.toxodb.org). The authors declare that all data generated supporting the findings of this study are available within the article and its supplementary information file. Plasmids, parasite and host cell strains (except for the mutant strain) are available from the corresponding author upon request.

\section{Competing interests}

The authors declare that they have no competing interests.

\section{Funding}

The study was funded by the CSU East Bay Faculty Support Grant (PSG), CSU East Bay Center for Student Research Student Supply Grants (EVQ), and California State University Program for Education and Research Biotechnology (CSUPERB) New Investigator Award (PSG).

\section{Authors' Contributions}

Conceptualization: EVQ, PSG. Data collection: EVQ, BC, DH, JS. Data curation: EVQ, EB, BC PSG. Formal analysis: EVQ, EB, BC, PSG. Writing original manuscript: EVQ, EB, PSG. Manuscript editing and revision: PSG. Supervision: PSG. All authors have read and approved the final manuscript.

\section{Acknowledgements}


The authors thank John Boothroyd for providing the parental Toxoplasma strain, plasmids, and antibodies used in this study.

\section{References}

1. Aguirre AA, Longcore T, Barbieri M, Dabritz H, Hill D, Klein PN, et al. The One Health Approach to Toxoplasmosis: Epidemiology, Control, and Prevention Strategies. Ecohealth. 2019;16:378-90.

2. Dubey JP. Bradyzoite-induced murine toxoplasmosis: stage conversion, pathogenesis, and tissue cyst formation in mice fed bradyzoites of different strains of Toxoplasma gondii. J Eukaryot Microbiol. 1997;44:592-602.

3. Jeffers V, Tampaki Z, Kim K, Sullivan WJ. A latent ability to persist: differentiation in Toxoplasma gondii. Cell Mol Life Sci CMLS. 2018;75:2355-73.

4. Dubey JP, Lindsay DS, Speer CA. Structures of Toxoplasma gondii Tachyzoites, Bradyzoites, and Sporozoites and Biology and Development of Tissue Cysts. Clin Microbiol Rev. 1998;11:267-99.

5. Coppin A, Dzierszinski F, Legrand S, Mortuaire M, Ferguson D, Tomavo S. Developmentally regulated biosynthesis of carbohydrate and storage polysaccharide during differentiation and tissue cyst formation in Toxoplasma gondii. Biochimie. 2003;85:353-61.

6. Guérardel Y, Leleu D, Coppin A, Liénard L, Slomianny C, Strecker G, et al. Amylopectin biogenesis and characterization in the protozoan parasite Toxoplasma gondii, the intracellular development of which is restricted in the HepG2 cell line. Microbes Infect Inst Pasteur. 2005;7:41-8.

7. Uboldi AD, McCoy JM, Blume M, Gerlic M, Ferguson DJP, Dagley LF, et al. Regulation of Starch Stores by a $\mathrm{Ca}(2+)$-Dependent Protein Kinase Is Essential for Viable Cyst Development in Toxoplasma gondii. Cell Host Microbe. 2015;18:670-81.

8. Sugi T, Tu V, Ma Y, Tomita T, Weiss LM. Toxoplasma gondii Requires Glycogen Phosphorylase for Balancing Amylopectin Storage and for Efficient Production of Brain Cysts. mBio. 2017;8:e01289-17.

9. Ray WJ, Peck EJ. 12 Phosphomutases. In: Boyer PD, editor. The Enzymes. Academic Press; 1972. p. 407-77.

10. ToxoDB. https://toxodb.org/toxo/app. Accessed 17 Dec 2021.

11. Matthiesen SH, Shenoy SM, Kim K, Singer RH, Satir BH. A parafusin-related Toxoplasma protein in Ca2+-regulated secretory organelles. Eur J Cell Biol. 2001;80:775-83.

12. Pittman KJ, Aliota MT, Knoll LJ. Dual transcriptional profiling of mice and Toxoplasma gondii during acute and chronic infection. BMC Genomics. 2014;15:806.

13. Matthiesen SH, Shenoy SM, Kim K, Singer RH, Satir BH. Role of the parafusin orthologue, PRP1, in microneme exocytosis and cell invasion in Toxoplasma gondii. Cell Microbiol. 2003;5:613-24.

14. Liu L, Tucker SC, Satir BH. Toxoplasma PRP1 is an ortholog of parafusin (PFUS) in vesicle scaffold assembly in Ca2+-regulated exocytosis. Eur J Cell Biol. 2009;88:301-13.

15. Saha S, Coleman BI, Dubey R, Blader IJ, Gubbels M-J. Two Phosphoglucomutase Paralogs Facilitate Ionophore-Triggered Secretion of the Toxoplasma Micronemes. mSphere. 2017;2:e00521-17. 
16. Shen B, Brown KM, Lee TD, Sibley LD. Efficient gene disruption in diverse strains of Toxoplasma gondii using CRISPR/CAS9. mBio. 2014;5:e01114-01114.

17. Soldati D, Boothroyd JC. Transient transfection and expression in the obligate intracellular parasite Toxoplasma gondii. Science. 1993;260:349-52.

18. Weiss LM, Laplace D, Takvorian PM, Tanowitz HB, Cali A, Wittner M. A cell culture system for study of the development of Toxoplasma gondii bradyzoites. J Eukaryot Microbiol. 1995;42:150-7.

19. Sharma J, Rodriguez P, Roy P, Guiton PS. Transcriptional ups and downs: patterns of gene expression in the life cycle of Toxoplasma gondii. Microbes Infect. 2020;22:525-33.

20. Abdelbaset AE, Fox BA, Karram MH, Ellah MRA, Bzik DJ, Igarashi M. Lactate dehydrogenase in Toxoplasma gondii controls virulence, bradyzoite differentiation, and chronic infection. PLOS ONE. 2017;12:e0173745.

21. Mouveaux T, Oria G, Werkmeister E, Slomianny C, Fox BA, Bzik DJ, et al. Nuclear Glycolytic Enzyme Enolase of Toxoplasma gondii Functions as a Transcriptional Regulator. PLOS ONE. 2014;9:e105820.

22. Garfoot AL, Cervantes PW, Knoll LJ. Transcriptional Analysis Shows a Robust Host Response to Toxoplasma gondii during Early and Late Chronic Infection in Both Male and Female Mice. Infect Immun. 2019. https://doi.org/10.1128/IAl.00024-19.

23. Sidik SM, Huet D, Ganesan SM, Huynh M-H, Wang T, Nasamu AS, et al. A Genome-wide CRISPR Screen in Toxoplasma Identifies Essential Apicomplexan Genes. Cell. 2016;166:1423-1435.e12.

24. Waldman BS, Schwarz D, Wadsworth MH, Saeij JP, Shalek AK, Lourido S. Identification of a Master Regulator of Differentiation in Toxoplasma. Cell. 2020;180:359-372.e16.

25. Imada M, Kawashima S, Kanehisa M, Takeuchi T, Asai T. Characterization of alphaphosphoglucomutase isozymes from Toxoplasma gondii. Parasitol Int. 2010;59:206-10.

26. Levin S, Almo SC, Satir BH. Functional diversity of the phosphoglucomutase superfamily: structural implications. Protein Eng Des Sel. 1999;12:737-46.

27. Shukla A, Olszewski KL, Llinás M, Rommereim LM, Fox BA, Bzik DJ, et al. Glycolysis is important for optimal asexual growth and formation of mature tissue cysts by Toxoplasma gondii. Int $\mathrm{J}$ Parasitol. 2018;48:955-68.

\section{Figures}




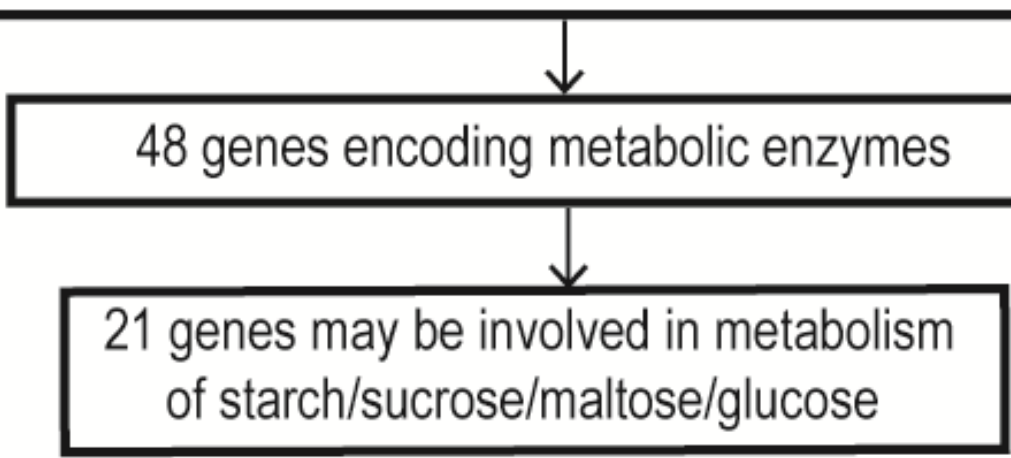

$\mathrm{B}$

starch degradation III heterolactic fermentation glycogen degradation I sucrose biosynthesis II superpathway of anaerobic sucros. starch degradation V starch biosmithesis Glycolysis / Gluconeogenesis

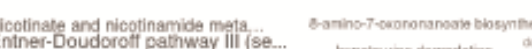

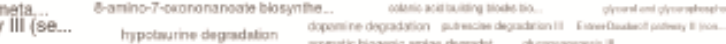

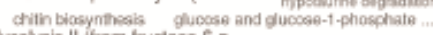
gycolysis II ifrom fructose 6.p.

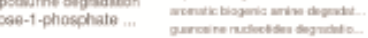
ganasion I

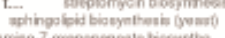

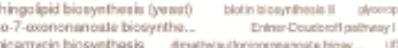

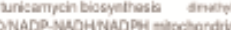

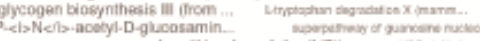

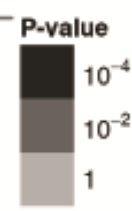

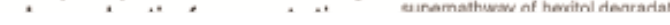

\section{Figure 1}

Identification of upregulated metabolic genes during Toxoplasma chronic infection (A) Workflow for identification of genes associated with glycolysis and gluconeogenesis with higher expression in chronic vs. acute infection in dataset from Pittman et al.[12]; the analysis was performed on ToxoDB [10]. (B) Word cloud of enriched pathways among the 422 genes upregulated during chronic infection in mice. The image was generated on ToxoDB. (C) Transcript levels of differentially regulated glycolytic and 
gluconeogenic enzymes in Toxoplasma. Values were obtained from Pittman et al. dataset available on ToxoDB version 54.

\section{Figure 2}

Disruption of pgm1 and growth assays. (A) Schematic representation of disruption of pgm1 using CRISPR-Cas 9 gene-editing system for nonhomologous insertion of the hxgprt selectable marker cassette. The dotted line represents the region in the first exon of $p g m 1$ targeted by the small guide RNA (sgPGM1). (B) Image of DNA gel electrophoresis of PCR1-3 performed using DNA from wildtype (WT) and mutant $(\triangle p g m 1)$ to demonstrate integration of the hxgprt expression cassette at the $p g m 1$ locus. The expected product for PCR1 (212bp) was obtained only for WT while products for PCR2 (813bp) and PCR3 (1185bp) were amplified only with $\triangle p g m 1$ DNA. (C) Intracellular growth. HFFs were infected with $1.2 \mathrm{x}$ $10^{5}$ WT or $\triangle p g m 1$ parasites for 24 hours in cDMEM. Monolayers were fixed and stained with antibodies raised against SAG1 (tachyzoite surface marker) and GRA7 (PV marker). Intracellular parasites were enumerated in at least 20 vacuoles/strain/experiment, $\mathrm{N}=3$ independent experiments; error bars = standard error of the mean; $p$-value was determined by Chi-square test. (D) Total numbers of plaques counted 10 days after infection of HFFs with 250 WT or $\Delta p g m 1$ parasites. (E) Plaque areas were determined for 85 WT and $109 \Delta p g m 1$ plaques using Fiji/Image in pixels $^{2}, \mathrm{~N}=3$ replicates/strain in a single experiment, error bar $=$ standard deviation; $n s: ~ p$-value $>0.05$ by nonparametric Mann-Whitney test.

\section{Figure 3}

In vitro stage conversion assay. (A) Representative fluorescence images of amylopectin-containing WT and $\Delta p g m 1$ cysts at 4 days post-induction. Infected monolayers were stained with PAS to detect amylopectin (red), DBA to label the cyst wall (green), and DAPI for nuclei (blue); Scale bar $=10$ microns. (B) Representative images of WT and $\Delta p g m 1$ cysts 4 days post-induction in vitro. The images are representative of the mean value of cyst areas for each strain. Cysts were stained with DBA (red), antiGRA7 (green), and DAPI (blue); Scale bar = 10 microns. (C) Quantification of cyst sizes. The areas of 176 WT and $185 \Delta p g m 1$ cysts were determined in pixels ${ }^{2}$ at 4 days post-induction from 3 independent experiments; ${ }^{*} p=0.0362$ by nonparametric Mann-Whitney test.

\section{Supplementary Files}

This is a list of supplementary files associated with this preprint. Click to download.

- AdditionalFile1.pdf 